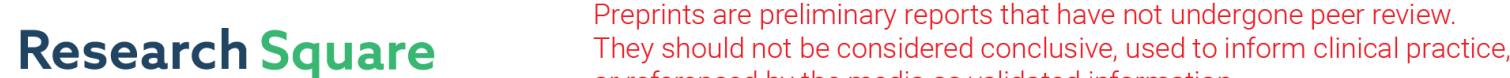 or referenced by the media as validated information. \\ Long Noncoding RNA SNHG10 Inhibits Renal Fibrosis By Negatively Regulating MiR-378b Expression
}

\section{Jian Hao}

the Third Clinical Medical College of Shanxi Medical University

Yun Zhou ( $\square$ zhouyun_sx@163.com)

Shanxi Provincial People's Hospital

\section{Weimin Yu}

the Third Clinical Medical College of Shanxi Medical University

Hui Li

the Third Clinical Medical College of Shanxi Medical University

Dandan He

the Third Clinical Medical College of Shanxi Medical University

\section{Research Article}

Keywords: LncRNA SNHG10, MiR-378b, Proliferation, Extracellular matrix, Renal fibrosis.

Posted Date: April 13th, 2021

DOI: https://doi.org/10.21203/rs.3.rs-410408/v1

License: (c) (i) This work is licensed under a Creative Commons Attribution 4.0 International License. Read Full License 


\section{Abstract}

Background: LncRNA have been increasingly shown that plays pivotal roles in the development of various diseases, including renal fibrosis. Nevertheless, the pathological function of Long non-coding RNA SNHG10 (SNHG10) in the renal fibrosis remains obscure.

Methods: We detected the expression levels of SNHG10 in the tissue samples and cell lines via RT-qPCR analysis. The functions of SNHG10 on the progression of renal fibrosis were examined by CCK-8, EdU, dual luciferase reporter and immunofluorescence analyses.

Results: In the present study, SNHG10, production of extracellular matrix (ECM), including a-SMA and Fibronectin levels were significantly increased in HK-2 cells after TGF- $\beta$ stimulation. Ectopic of SNHG10 inhibited cell proliferation and inhibits thea-SMA and Fibronectin expression of TGF- $\beta 1$-induced HK-2 cells. In addition, bioinformatics analysis and dual luciferase reporter assay indicated that miR-378b was a target gene of SNHG10. Mechanistically, miR-378b overexpression abolished the repressive effects of SNHG10 on TGF- $\beta 1$-induced HK-2 cells.

Conclusion: SNHG10 plays an anti-fibrotic effect through suppression of miR-378b expression in renal fibrosis, which provides a promising therapeutic target for the treatment of renal fibrosis.

\section{Introduction}

Chronic kidney disease (CKD) is a type of progressive diseases and a critical global public health issue which leading contributions to death globally with a prevalence rate of $10 \%$ to $12 \%$ in the world [1-4]. Owing to lack of renal reserve function and clinical features in the early onset, CKD generally progresses to the middle and late stages when obvious symptoms were appeared [5]. Fibrosis is a hallmark and common pathway leading to end-stage organ dysfunction [6]. Renal interstitial fibrosis, as the final pathological hallmark of CKD, irreversibly damages of renal function, and the final convergent pathway of renal disease [7-9].

Renal fibrosis is the usually ultimate manifestation of various kidney diseases including glomerulonephritis and diabetic nephropathy [10]. In histological features, renal fibrosis manifests the excessive deposition of extracellular matrix (ECM) [11], which is always companied by various pathological alterations in tubular epithelial cells including epithelial-to-mesenchymal transition (EMT), fibroblast activation, immune cell infiltration, and apoptosis $[12,13]$.

Despite that extensive research exists on the pathogenesis and molecular mechanisms underlying of renal fibrosis, the effective intervention for this disease is still lacking. In view of that renal fibrosis can lead to scar formation and even renal failure, further exploring the novel mechanisms of renal fibrosis, as well as searching for novel CKD therapeutic targets is compellingly needed, which is of great significance for the development of effective therapeutic strategies and prevention of patients with renal diseases. 
Long non-coding RNAs (IncRNAs) are a type of RNAs whose transcripts are more than 200 nucleotides in length, and have no/little potentials in coding proteins [14, 15]. A growing body of evidence indicates that IncRNAs play extensive regulatory roles in life activities and the development of many diseases and exert vital roles in the regulation of cell proliferation, migration, invasion and apoptosis [16-19]. In recent years, researchers have proved IncRNAs act as important regulators in the progress of fibrosis. For instance, silencing Inc-Hser aggravated liver fibrosis through inducing the epithelial-mesenchymal transition (EMT) and apoptosis[20]. Liu et al proved that Inc-PCF can accelerate pulmonary fibrogenesis by directly targeting miR-344a-5p to regulate map3k11[21]. Moreover, study has demonstrated that the liver-enriched IncRNA Lfar1 could promote hepatic fibrosis through inducing hepatic stellate cells activation and hepatocytes apoptosis [22]. These findings showed that IncRNAs played an important role in fibrosis progression. However, to date, the roles of IncRNA SNHG10 in renal fibrosis progression was not illuminated.

In this study, experiments were implemented to shed light on the potential role of SNHG10 in renal fibrosis progression, providing novel prognostic biomarkers for the development of patients with renal diseases.

\section{Materials And Methods}

\section{Cell culture and treatment}

Human nephric proximal tubular epithelial cell line HK-2 was purchased from American Tissue Culture Collection (ATCC; Rockville, USA) and grown in DMEM complemented with $10 \% \mathrm{FBS}$ with $5 \% \mathrm{CO}_{2}$ in an incubator at $37^{\circ} \mathrm{C}$. Cells were starved for $24 \mathrm{~h}$ before any stimulation. For establishment of renal fibrosis, HK-2 cells were stimulated with different doses of transforming growth factor $\beta 1$ (TGF- $\beta 1$ ) for different hours and then subjected to further analysis.

\section{Cell transfection}

The mimic of miR-378b as well as negative control (NC mimic) were purchased from Invitrogen (Carlsbad, CA, USA). The full length of SNHG10 was ligated in pcDNA3.1 vector (pcDNA3.1 SNHG10) was purchased from GenePharma (Shanghai, China) with empty plasmid as negative control (pcDNA3.1). Cell transfection was conducted using Lipofectamine 2000 (Thermo Fisher Scientific, Waltham, MA, USA). After $48 \mathrm{~h}$, cells were harvested for following experiments.

\section{Quantitative real-time PCR (qRT-PCR) ananlysis}

The total RNA from cells was extracted with Trizol reagent (Invitrogen) and subsequently reversed transcribed into cDNA by utilizing PrimeScript RT reagent Kit (Takara, Ohtsu, Japan). Real-time PCR was carried out with SYBR Premix EX Taq ${ }^{\text {TM }}$ II kit (TaKaRa, Dalian, China) on the PCR detection instrument (Opticon CFD-3200; MJ Research, Waltham, MA, USA). U6 and GAPDH were used as internal reference. The quantification of gene relative expression analysis was carried out using the $2^{-\triangle \triangle C t}$ method. The primer sequences for PCR were displayed as follow: SNHG10: 5'-GTTGGTCTCTTGGGAGGTAG-3' 
(forward) and 5'-CGCCACGACGAACTGCATGC-3' (reverse); miR-378b: 5'-CCACGACCATCATCAGGTGAA-3' (forward) and 5'-CCTCACGGAGGCATTCTAAAGT-3' (reverse); GAPDH: 5'-TATGATGATATCAAGAGGGTAGT3' (forward) and 5'-TGTATCCAAACTCATTGTCATAC-3' (reverse); U6: 5'-CTCGCTTCGGCAGCACA-3' (forward) and 5'-AACGCTTCACGAATTTGCGT-3' (reverse).

\section{Western blot assay}

Cells were lysed with RIPA Buffer (cat: 89900; Thermo Fisher Scientific, USA), and the protein concentration was detected with the BCA Kit (cat: P0012S; Beyotime, Beijing, China). The equivalent amount of proteins were segregated on $12 \%$ SDS-PAGE and subsequently transferred to PVDF membranes (Millipore, Billerica, MA, USA). Blocking the film with BSA and sealed with $5 \%$ skim milk, then the membranes was incubated with the primary antibody at $4{ }^{\circ} \mathrm{C}$ overnight, followed by incubation with secondary antibody at room temperature for $1 \mathrm{~h}$. Finally, the bands were measured with enhanced chemiluminescence reagent ECL detection kit (Thermo Fisher Scientifc, Inc.) on ChemiDoc XRS System (Bio-Rad, Hercules, CA, USA).

\section{Cell proliferation assay}

Cell visibility was assessed using the Cell Counting Kit-8 (CCK-8) assay. In details, cells were plated in 96well plates at a density of $1 \times 10^{5}$ cells/well and cultivated at $37^{\circ} \mathrm{C}$ in the presence of $5 \% \mathrm{CO}_{2}$. At $0,24,48$ and $72 \mathrm{~h}$ post incubation, cells were processed with CCK-8 reagent (Dojindo, Tokyo, Japan), followed by incubation for additional $4 \mathrm{~h}$ at $37^{\circ} \mathrm{C}$. At last, absorbance was examined at wave length of $450 \mathrm{~nm}$ by a microplate reader (BioTek, Winooski, VT, USA).

\section{Immunofluorescence assay}

Transfected cells were cultured for $24 \mathrm{~h}$ at $37^{\circ} \mathrm{C}$ on a Petri dish and fixed in $4 \%$ formaldehyde. After PBS washes, cells were penetrated and blocked with $5 \%$ BSA for $2 \mathrm{~h}$. Then, the cells were incubated with the primary antibody overnight at $4^{\circ} \mathrm{C}$ and then incubated with the secondary antibody for $30 \mathrm{~min}$. DAPI (1: 1000 ) was used to stain the nuclei. The laser scanning fluorescence microscope was used to visualize fluorescence.

\section{Luciferase reporter assay}

The WT and MUT 3'-UTR sequences of SNHG10 were synthesized by Sangon Biotech. The 3'UTR of SNHG10 containing or without binding sites for miR-378b were cloned into pGL3 reporter vector (Promega, Madison, WI, USA) to shape SNHG10-WT or SNHG10-MUT, respectively. Cells were cotransfected with indicated vectors and miR-378b mimic or NC mimic by using Lipofectamine 2000 (Thermo Fisher Scientific, Waltham, MA, USA) according to the product instructions. After $48 \mathrm{~h}$, the luciferase activity was examined using Dual-Luciferase Reporter Assay System (Promega).

\section{Statistical analysis}

All data were shown as means \pm SD via three parallel experiments. Statistical analysis was conducted by employment of SPSS 21.0 software. Student's $t$ test and one-way ANOVA were applied for comparison 
between two or more groups. Differences were defined as statistically significant when $\mathrm{P}<0.05$.

\section{Results}

\section{The SNHG10 expression was decreased in TGF- $\beta$-induced HK-2 cells}

We investigated the role of SNHG10 in renal fibrosis using HK-2 cells in vitro. After incubation with the pro-fibrotic cytokine TGF- $\beta 1$, the expression of SNHG10 was markedly decreased at $48 \mathrm{~h}$ and $72 \mathrm{~h}$ (Fig. 1A). Moreover, the main components of ECM including a-SMA and Fibronectin were significantly induced in HK-2 cells after TGF- $\beta$ stimulation (Fig. 1B, C). These results potentially indicated that SNHG10 plays a protective role in attenuating renal fibrosis.

\section{Ectopic of SNHG10 inhibited cell proliferation of TGF- $\beta 1-$ induced HK-2 cells}

In order to investigate the effects of SNHG10 on renal fibrosis in vitro, HK-2 cells were transfected with pcDNA3.1 SNHG10 and negative control, and qRT-PCR assay was performed to detect the transfected efficiency. The results certified that pcDNA3.1 SNHG10 led to overexpression of SNHG10 in TGF- $\beta 1$ induced HK-2 cells compared to the pcDNA3.1 group (Fig. 2A). Moreover, we explore the effect of SNHG10 on the viability of TGF- $\beta 1$-induced HK-2 cells by the CCK-8 assay. The data of Fig. $2 \mathrm{~B}$ demonstrated that overexpression of SNHG10 remarkably suppressed the viability of HK-2 cells, when compared with pcDNA3.1 group. Subsequently, the role of SNHG10 in the proliferation of TGF- $\beta 1$-induced HK-2 cells was determined by colony formation assay. The results verified that transfection with pcDNA3.1 SNHG10 significantly suppressed the proliferation of TGF- $\beta 1$-induced HK-2 cells (Fig. 2C). The data of EdU assay revealed that the EdU-positive cells in HK-2 cells transfected with pcDNA3.1 SNHG10 were obviously reduced compared to pcDNA3.1 group (Fig. 2D). In addition, western blot assay was carried out to assess the effects of SNHG10 on the cell proliferation-related proteins. Up-regulating SNHG10 dramatically suppressed the expression levels of Ki-67 and PCNA in TGF- $\beta 1$-induced HK- 2 cells (Fig. 2E).

\section{Overexpression of SNHG10 inhibits the expression of $a-$ SMA and Fibronectin in TGF- $\beta 1$-induced HK-2 cells}

To further explore the roles of SNHG10 in the renal fibrosis, the qRT-PCR was applied to detect the relative expression of a-SMA, a marker of myofibroblasts, and Fibronectin, the major components of ECM. The results of Fig. 3A shown that the expression level of a-SMA and fibronectin were augmented caused by TGF- $\beta 1$ treatment and decreased by transfecting with pcDNA3.1 SNHG10 in HK-2 cells. Meanwhile, western blot analysis manifested that ectopic of SNHG10 aggravated the diminution of protein levels of a-SMA and Fibronectin which increased by TGF- $\beta 1$ administration (Fig. 3B). 


\section{MiR-378b is a direct target of SNHG10 in renal fibrosis}

In order to study on the possible target genes of SNHG10 involved in the occurrence and progression of renal fibrosis, we used Starbase to search for genes that were directly regulated by SNHG10. Among all the targets, miR-378b was chosen for further study, since miR-378 functions as an important regulator controlling the occurrence and development of various fibrosis. MiR-378 has been proved that miR-378 could limit activation of hepatic stellate cells and liver fibrosis[23]. Furthermore, miR-378 reduces mesangial hypertrophy and kidney tubular fibrosis[24]. The binding site between SNHG10 and miR-378b were as shown in Fig. 4A. In addition, the miR-378b expression in TGF- $\beta$-induced HK-2 cells was analyzed from $0 \mathrm{~h}$ to $72 \mathrm{~h}$ and results indicated that the expression of miR-378b was significantly increased at $48 \mathrm{~h}$ and $72 \mathrm{~h}$ (Fig. 4B). Furthermore, we found that miR-378b was negatively regulated by SNHG10. The expression level of miR-378b was markedly decreased in pcDNA3.1 SNHG10 group compared with pcDNA3.1 group (Fig. 4C).

To further validate whether SNHG10 binding to miR-378b, luciferase reporter assay was performed. The luciferase reporter plasmids were contained with the 3' UTR of SNHG10 wild type (WT) or mutant (Mut). As shown in Fig. 4D, the luciferase activity of SNHG10-WT was significantly decreased when cotransfected with miR-378b mimic, while no significant alterations were observed mutation at miR-378b binding site in the $3^{\prime}$ UTR of SNHG10.

\section{SNHG10 inhibits renal fibrosis through suppression of miR- 378b expression}

To further investigate the role of SNHG10/miR-378b in the regulation of renal fibrosis, we artificially overexpressed miR-378b in HK-2 cells. RT-PCR assay disclosed that the expression of miR-378b was successfully enhanced by transfected with miR-378b mimic (Fig. 5A). To confirm whether the effect of SNHG10 on renal fibrosis was mediated by miR-378b, miR-378b mimic was co-transfected with SNHG10 overexpressed in HK-2 cells (TGF- $\beta 1+$ pcDNA3.1 SNHG10 + miR-378b mimic). Proliferative capacity was determined by CCK- 8 and colony formation assays. The data displayed that miR-378b mimic abolished the repressive effects of SNHG10 overexpression on TGF- 31 -induced HK-2 cell viability (Fig. 5B and 5C). Consistently, the numbers of EdU-positive cells were increased correspondingly to the pcDNA3.1 SNHG10 + miR-378b mimic-transfected HK-2 cells compared with those in pcDNA3.1 SNHG10 group (Fig. 5D). Additionally, qRT-PCR and western blot assays illustrated that miR-378b mimic abolished the inhibitory effects of SNHG10 overexpression on the mRNA and protein expression levels of a-SMA and Fibronectin in TGF- $\beta 1$-induced HK-2 cells (Fig. 6).

\section{Discussion}

Renal fibrosis is increasingly becoming a major public health issue and considered as the common final stage of progressive renal disease [25]. The pathogenesis of renal fibrosis is a progressive process that reduces the capacity for tissue repair and ultimately leads to end-stage kidney failure $[13,26]$. 
Mounting evidence has emphasized that aberrant expression levels of IncRNAs and miRNAs were contributed to the kidney diseases including renal fibrosis $[27,28]$. Studies have demonstrated that ectopic the expression of IncRNA-SARCC could decrease RCC cells resistance to Sunitinib [29]. Wang et al proved that knockdown of IncRNA GAS5 contributed to anti-fibrosis by competitively binding miR-96-5p [30]. Moreover, IncRNA NR_038323 is also revealed to suppress HG-induced renal fibrosis through the miR-324-3p/DUSP1 axis [31]. Besides, IncRNA-LET serves a tumor suppressive role by regulating miR373-3p in RCC [32].

As a typical multifunctional IncRNA, SNHG10 is reported widely expressed in various diseases and plays a crucial role in multiple molecular and cellular processes. For instance, SNHG10 regulates HCC cell proliferation, invasion and epithelial-mesenchymal transition of by regulating SOX9 [33]. He et al also found that SNHG10 increases the methylation of miR-218 to facilitate cell proliferation in osteosarcoma [34]. However, explorations about the exact role of SNHG10 in the progression of renal fibrosis are scanty. Based on the literature, we hypothesize that SNHG10 may play regulatory function in the progression of renal fibrosis.

TGF- $\beta 1$ is an important factor responsible for renal fibrosis. Herein, we used HK-2 cells induced with TGF$\beta 1$ to establish the cellular renal fibrosis model in vitro. In the present study, we concentrated on the potential function of SNHG10 and further investigated its latent molecular mechanism in the renal fibrosis progression. We found that the expression level of miR-542-3p was overtly down-regulated in TGF- $\beta$-induced HK-2 cells.

Mounting evidence has emphasized that the main pathological change of renal fibrosis is the deposition of ECM and TGF- $\beta 1$-activated lung fibroblasts conceivably overlap with a-SMA positive myofibroblasts, which is responsible for producing ECM components, such as Fibronectin [35, 36]. Next, we performed gain-of-function experiments identified that ectopic of SNHG10 expression inhibits the proliferation and EMC deposition including a-SMA and Fibronectin in TGF- $\beta 1$-induced HK-2 cells. Meanwhile, miR-378b is a direct target of SNHG10 in renal fibrosis by using multiple bioinformatic analyses. These results provided evidence that SNHG10 may function as a protector in renal fibrosis progression through targeting miR$378 b$.

In conclusion, our study uncovered the first clue that SNHG10 plays an anti-fibrotic effect through the suppression of cell proliferation and down-regulation of a-SMA and Fibronectin expression by directly targeting miR-378b. On the whole, these findings provide a deep insight into the mechanisms underlying renal fibrosis, which developed a new therapeutic target for clinical prevention from devastating processes of renal fibrosis.

\section{Declarations}

Acknowledgements: We deeply appreciate the supports by all participants.

Conflict of interest: The authors state that there is no conflict of interest. 
Funding: Not applicable.

Ethics approval and consent to participate: Not applicable.

Consent for publication: Not applicable.

Authors' contributions: JH and DH participated in the design of the study and manuscript writing, WY performed the experiments, $\mathrm{HL}$ collected the data and performed the statistical analysis.

Data availability: The datasets used and/or analysed for the current study are available from the corresponding author upon reasonable request.

\section{References}

1. Lv W, Fan F, Wang Y, Gonzalez-Fernandez E, Wang C, Yang L, Booz GW, Roman RJ. Therapeutic potential of microRNAs for the treatment of renal fibrosis and CKD. Physiol Genom. 2018;50:20-34.

2. Gouveia MC, Vella JP, Cafeo FR, Affonso Fonseca FL, Bacci MR. Association between irisin and major chronic diseases: a review, European review for medical and pharmacological sciences, 20 (2016) 4072-4077.

3. Lv LL, Cao YH, Ni HF, Xu M, Liu D, Liu H, Chen PS, Liu BC. MicroRNA-29c in urinary exosome/microvesicle as a biomarker of renal fibrosis, American journal of physiology. Renal physiology. 2013;305:F1220-7.

4. Wen CP, Cheng TY, Tsai MK, Chang YC, Chan HT, Tsai SP, Chiang PH, Hsu CC, Sung PK, Hsu YH, Wen SF. All-cause mortality attributable to chronic kidney disease: a prospective cohort study based on 462293 adults in Taiwan, Lancet (London, England), 371 (2008) 2173-2182.

5. Gigante A, Di Mario F, Barbano B, Rosato E, Di Lazzaro Giraldi G, Pofi R, Gasperini ML, Amoroso D, Cianci R, Laviano A. Nutritional status and intrarenal arterial stiffness in cardiorenal syndrome: a pilot study. Eur Rev Med Pharmacol Sci. 2017;21:313-6.

6. Lin TA, Wu VC, Wang CY, Autophagy in Chronic Kidney Diseases, Cells, 8 (2019).

7. Zhou SG, Zhang W, Ma HJ, Guo ZY, Xu Y. Silencing of LncRNA TCONS_00088786 reduces renal fibrosis through miR-132, European review for medical and pharmacological sciences, 22 (2018) 166-173.

8. Chen Y, Mu L, Xing L, Li S, Fu S. Rhein alleviates renal interstitial fibrosis by inhibiting tubular cell apoptosis in rats. Biological research. 2019;52:50.

9. Bleyer AJ, Kidd K, Živná M, Kmoch S. Autosomal Dominant Tubulointerstitial Kidney Disease, Advances in chronic kidney disease, 24 (2017) 86-93.

10. Chevalier RL. The proximal tubule is the primary target of injury and progression of kidney disease: role of the glomerulotubular junction. Am J Physiol Renal Physiol. 2016;311:F145-61.

11. Olmos G, Muñoz-Félix JM, Mora I, Müller AG, Ruiz-Torres MP, López-Novoa JM. D. Rodríguez-Puyol, Impaired erythropoietin synthesis in chronic kidney disease is caused by alterations in extracellular 
matrix composition. J Cell Mol Med. 2018;22:302-14.

12. Liu Y. New insights into epithelial-mesenchymal transition in kidney fibrosis. Journal of the American Society of Nephrology: JASN. 2010;21:212-22.

13. Chang FC, Chou YH, Chen YT, Lin SL. Novel insights into pericyte-myofibroblast transition and therapeutic targets in renal fibrosis. Journal of the Formosan Medical Association = Taiwan yi zhi. 2012;111:589-98.

14. Robinson EK, Covarrubias S, Carpenter S. The how and why of IncRNA function: An innate immune perspective, Biochimica et biophysica acta. Gene regulatory mechanisms. 2020;1863:194419.

15. Zhang W, Tang G, Zhou S, Niu Y. LncRNA-miRNA interaction prediction through sequence-derived linear neighborhood propagation method with information combination. BMC Genomics. 2019;20:946.

16. Zhuo LA, Wen YT, Wang Y, Liang ZF, Wu G, Nong MD, Miao L. LncRNA SNHG8 is identified as a key regulator of acute myocardial infarction by RNA-seq analysis. Lipids Health Dis. 2019;18:201.

17. Wang L, Cho KB, Li Y, Tao G, Xie Z, Guo B. Long Noncoding RNA (IncRNA)-Mediated Competing Endogenous RNA Networks Provide Novel Potential Biomarkers and Therapeutic Targets for Colorectal Cancer, International journal of molecular sciences, 20 (2019).

18. Huang Z, Ye B, Wang Z, Han J, Lin L, Shan P, Cai X, Huang W. Inhibition of LncRNA-HRIM Increases Cell Viability by Regulating Autophagy Levels During Hypoxia/Reoxygenation in Myocytes, Cellular physiology and biochemistry: international journal of experimental cellular physiology, biochemistry, and pharmacology, 46 (2018) 1341-1351.

19. Han Y, Liu C, Lei M, Sun S, Zheng W, Niu Y, Xia X. LncRNA TUG1 was upregulated in osteoporosis and regulates the proliferation and apoptosis of osteoclasts. J Orthop Surg Res. 2019;14:416.

20. Zhang K, Zhang M, Yao Q, Han X, Zhao Y, Zheng L, Li G, Liu Q, Chang Y, Zhang P, Cui H, Shi Z, Chen T, Yao Z, Han T, Hong W. The hepatocyte-specifically expressed Inc-HSER alleviates hepatic fibrosis by inhibiting hepatocyte apoptosis and epithelial-mesenchymal transition. Theranostics. 2019;9:756682.

21. Liu H, Wang B, Zhang J, Zhang S, Wang Y, Zhang J, Lv C, Song X. A novel Inc-PCF promotes the proliferation of TGF- $\beta 1$-activated epithelial cells by targeting miR-344a-5p to regulate map3k11 in pulmonary fibrosis. Cell death disease. 2017;8:e3137.

22. Zhang K, Shi Z, Zhang M, Dong X, Zheng L, Li G, Han X, Yao Z, Han T, Hong W. Silencing IncRNA Lfar1 alleviates the classical activation and pyoptosis of macrophage in hepatic fibrosis. Cell death disease. 2020;11:132.

23. Hyun J, Wang S, Kim J, Rao K, Park S, Chung I, Ha C, Kim S, Yun Y. Y.J.N.c. Jung, MicroRNA-378 limits activation of hepatic stellate cells and liver fibrosis by suppressing Gli3 expression, 7 (2016) 10993.

24. Wang B, Yao K, Wise A, Lau R, Shen H, Tesch G, S.J.C.s. Ricardo, miR-378 reduces mesangial hypertrophy and kidney tubular fibrosis via MAPK signalling, 131 (2017) 411-423. 
25. Chen B. The miRNA-184 drives renal fibrosis by targeting HIF1AN in vitro and in vivo. Int Urol Nephrol. 2019;51:543-50.

26. Genovese F, Manresa AA, Leeming DJ, Karsdal MA, Boor P. The extracellular matrix in the kidney: a source of novel non-invasive biomarkers of kidney fibrosis? Fibrogenesis tissue repair. 2014;7:4.

27. Liu M, Ning X, Li R, Yang Z, Yang X, Sun S, Qian Q. Signalling pathways involved in hypoxia-induced renal fibrosis. J Cell Mol Med. 2017;21:1248-59.

28. Nogueira A, Pires MJ, Oliveira PA. Pathophysiological Mechanisms of Renal Fibrosis: A Review of Animal Models and Therapeutic Strategies, In vivo (Athens, Greece), 31 (2017) 1-22.

29. Zhai W, Sun Y, Guo C, Hu G, Wang M, Zheng J, Lin W, Huang Q, Li G, Zheng J, Chang C. LncRNASARCC suppresses renal cell carcinoma (RCC) progression via altering the androgen receptor(AR)/miRNA-143-3p signals. Cell death differentiation. 2017;24:1502-17.

30. Wang W, Jia YJ, Yang YL, Xue M, Zheng ZJ, Wang L, Xue YM, LncRNA GAS5 exacerbates renal tubular epithelial fibrosis by acting as a competing endogenous RNA of miR-96-5p, Biomedicine \& pharmacotherapy = Biomedecine \& pharmacotherapie, 121 (2020) 109411.

31. Ge Y, Wang J, Wu D, Zhou Y, Qiu S, Chen J, Zhu X, Xiang X, Li H, Zhang D. IncRNA NR_038323 Suppresses Renal Fibrosis in Diabetic Nephropathy by Targeting the miR-324-3p/DUSP1 Axis, Molecular therapy. Nucleic acids. 2019;17:741-53.

32. Ye Z, Duan J, Wang L, Ji Y, Qiao B. LncRNA-LET inhibits cell growth of clear cell renal cell carcinoma by regulating miR-373-3p. Cancer cell international. 2019;19:311.

33. Lan T, Yuan K, Yan X, Xu L, Liao H, Hao X, Wang J, Liu H, Chen X, Xie K, Li J, Liao M, Huang J, Zeng Y, Wu H. LncRNA SNHG10 Facilitates Hepatocarcinogenesis and Metastasis by Modulating Its Homolog SCARNA13 via a Positive Feedback Loop. Cancer research. 2019;79:3220-34.

34. He P, Xu Y, Wang Z. LncRNA SNHG10 increases the methylation of miR-218 gene to promote glucose uptake and cell proliferation in osteosarcoma. J Orthop Surg Res. 2020;15:353.

35. Pan R, Zhang Y, Zheng M, Zang B, Jin M. Hydroxysafflor Yellow A Suppresses MRC-5 Cell Activation Induced by TGF- $\beta 1$ by Blocking TGF- $\beta 1$ Binding to T $\beta R I I$. Front Pharmacol. 2017;8:264.

36. Bernard K, Logsdon NJ, Benavides GA, Sanders Y, Zhang J, Darley-Usmar VM, Thannickal VJ. Glutaminolysis is required for transforming growth factor- $\beta 1$-induced myofibroblast differentiation and activation. J Biol Chem. 2018;293:1218-28.

\section{Figures}


A

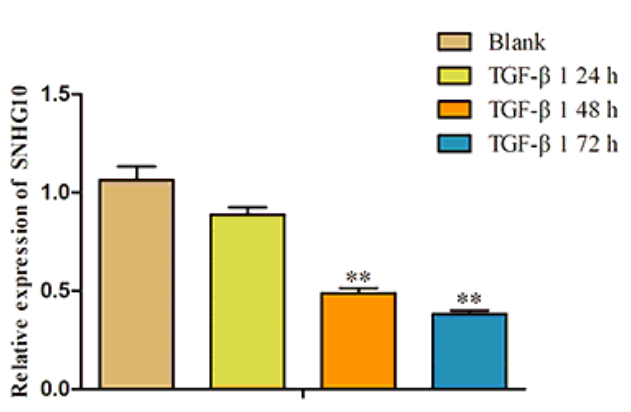

B

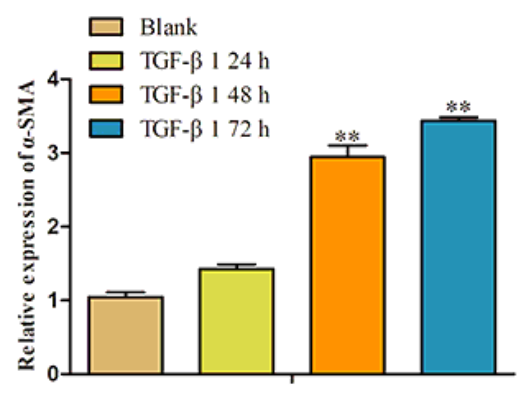

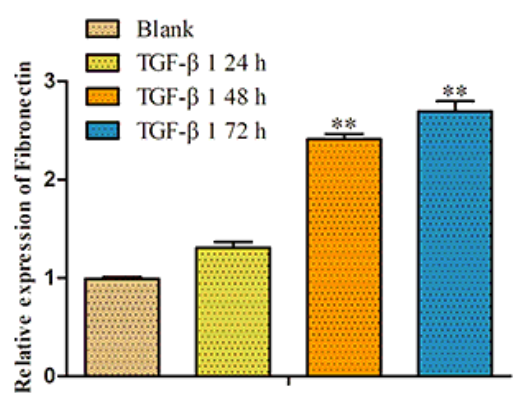

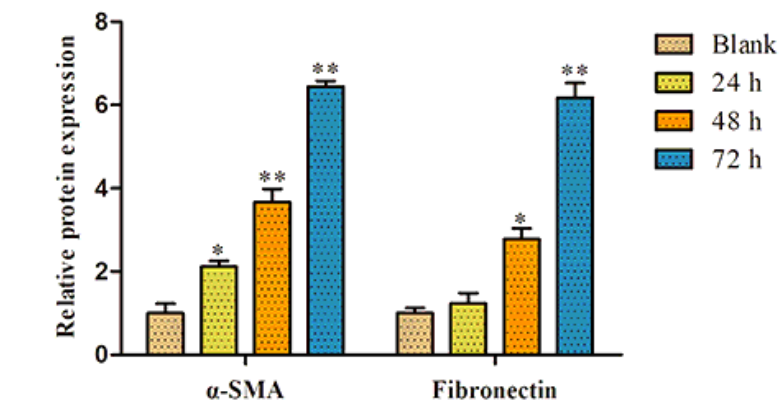

$\mathrm{C}$

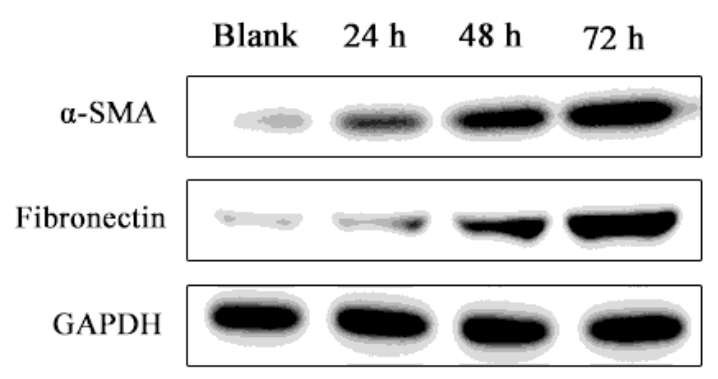

\section{Figure 1}

The SNHG10 expression was decreased in TGF- $\beta$-induced HK-2 cells. (A) The SNHG10 expression in TGF$\beta$-induced HK-2 cells from $0 \mathrm{~h}$ to $72 \mathrm{~h}$. $(B, C)$ The mRNA and protein expressions of $a-S M A$ and Fibronectin with treatment with TGF- $\beta(10 \mathrm{ng} / \mathrm{ml})$ was detected by RT-PCR and western blot analyses. ${ }^{\star} \mathrm{P}<0.05$, $\star \star \mathrm{P}<$ 0.01 vs. Blank. 
A

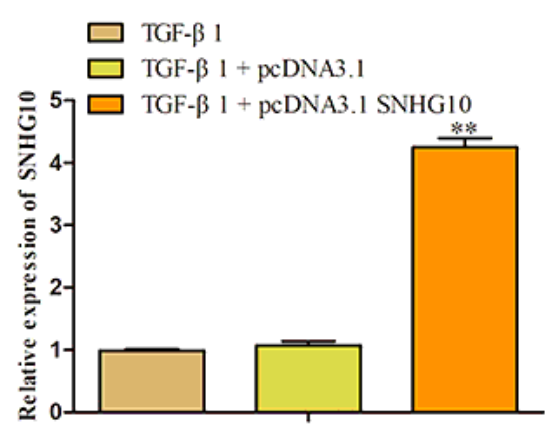

B

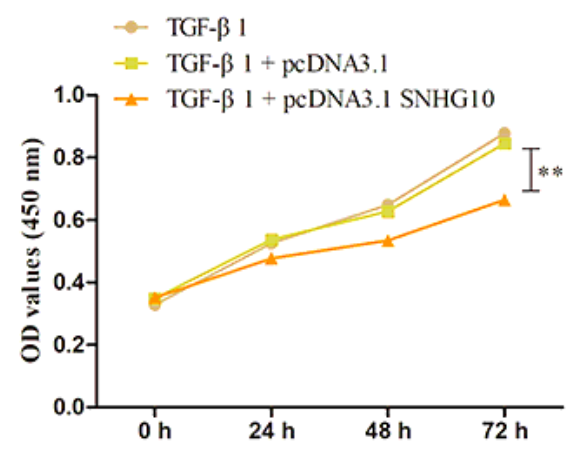

C TGF- $\beta 1$ TGF- $\beta 1+p c D N A 3.1$ TGF- $\beta 1+p c D N A 3.1$ SNHG10

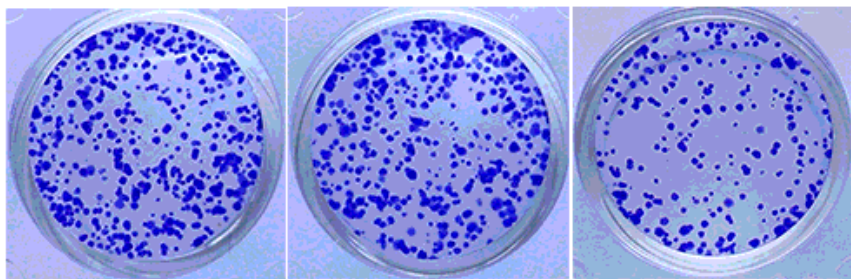

D TGF- $\beta 1 \quad T G F-\beta 1+p c D N A 3.1$ TGF- $\beta 1+p c D N A 3.1$ SNHG10
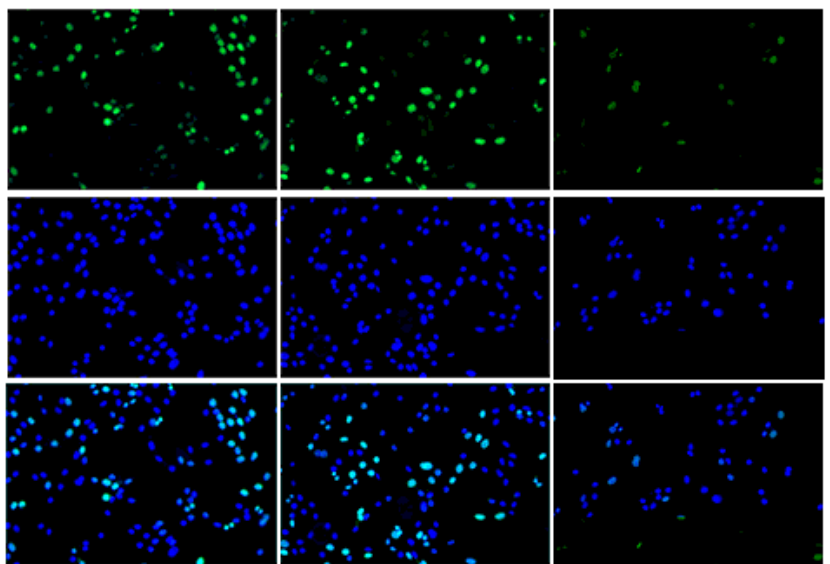

$\mathrm{E}$ $T G F-\beta 1 T_{T G F-\beta 1}+\underset{T G D N-\beta 1+p c D N A 3.1}{S N H G 10}$

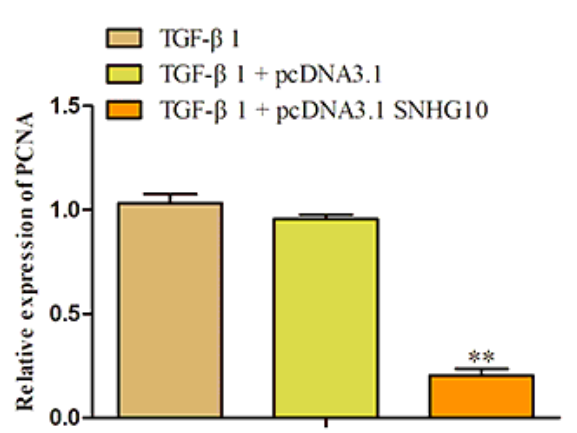

\section{Figure 2}

Ectopic of SNHG10 inhibited cell proliferation of TGF- $\beta 1$-induced HK-2 cells. (A) qRT-PCR assay was performed to detect the transfected efficiency. (B-D) CCK-8, colony formation and EdU assays were used to assess the proliferation ability of HK-2 cells. (E) The expression levels of PCNA and Ki-67 were analyzed by western blot assay. ${ }^{\star *} \mathrm{P}<0.01$ vs. TGF- $\beta 1$. 


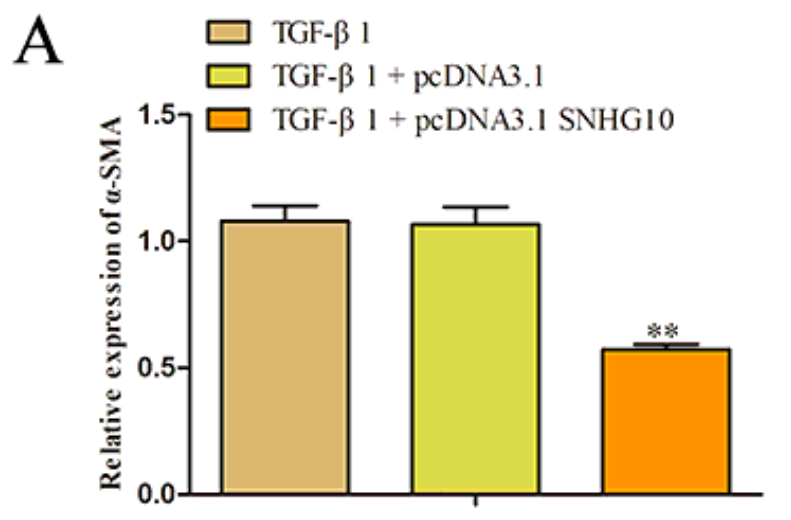

B
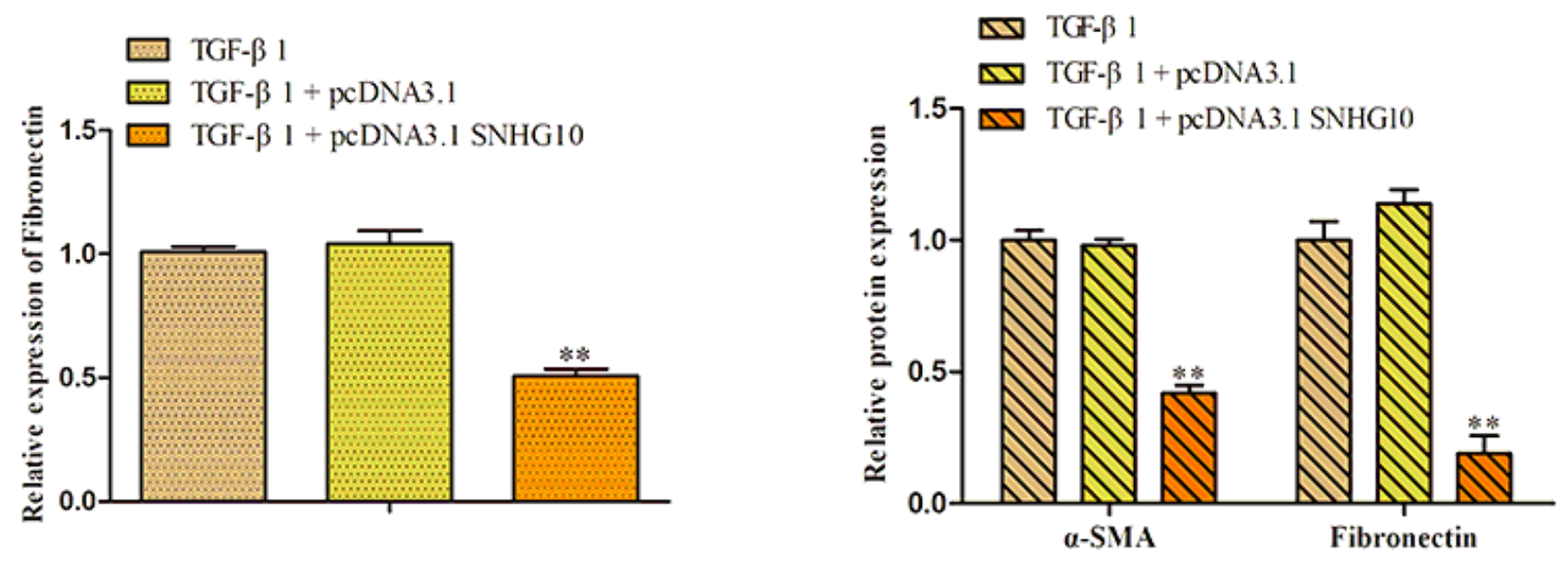

Figure 3

Overexpression of SNHG10 inhibits the expression of a-SMA and Fibronectin in TGF- $\beta 1$-induced HK-2 cells. $(A, B)$ The relative mRNA and protein expression of a-SMA and Fibronectin were analyzed by using qRT-PCR and western blote analyses. ${ }^{*} \mathrm{P}<0.01$ vs. TGF- $\beta 1$. 
B $\square$ Blank

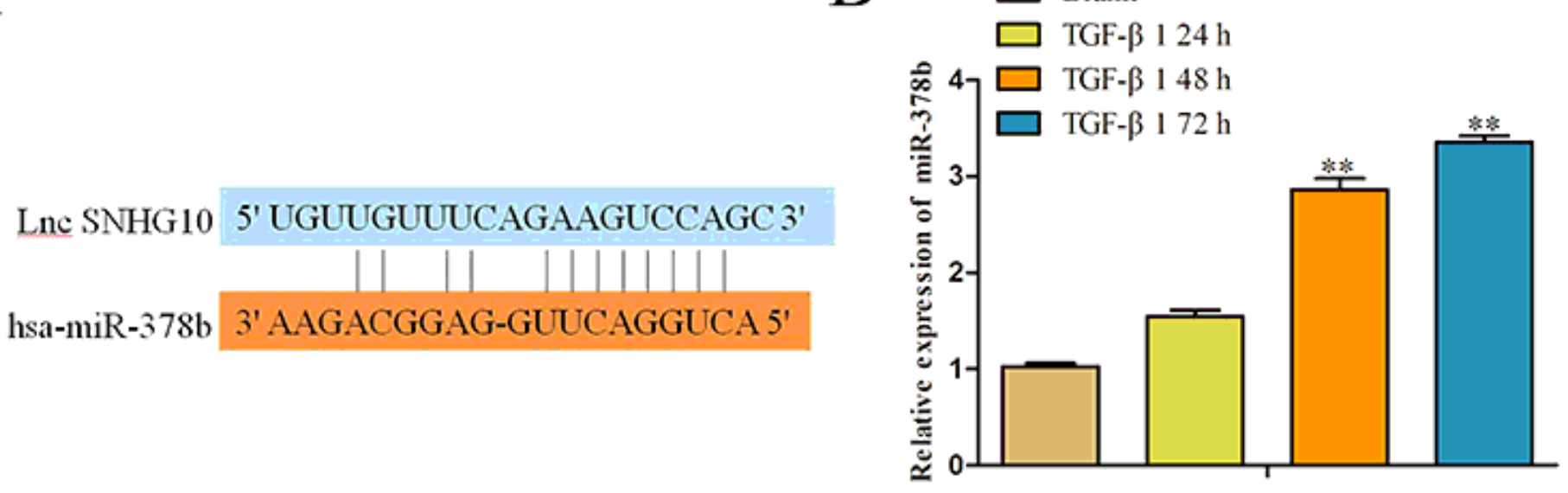

$\mathrm{C}$

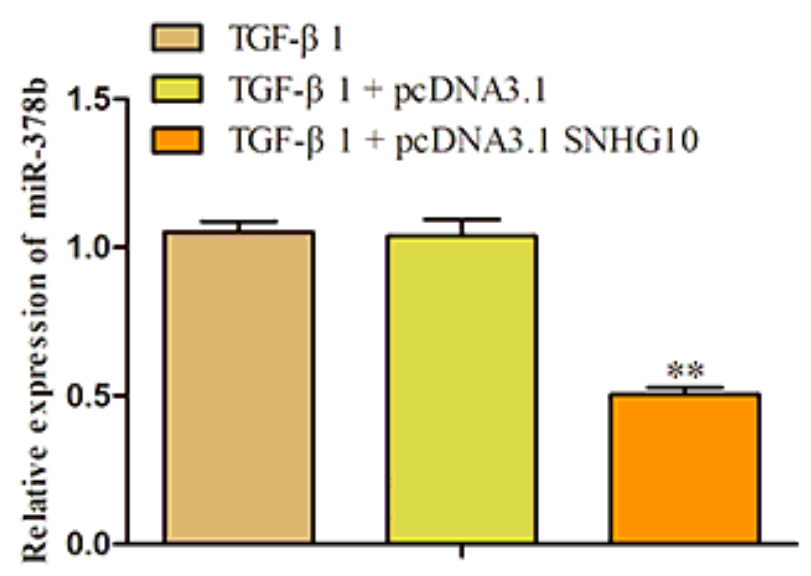

$\mathrm{D}$

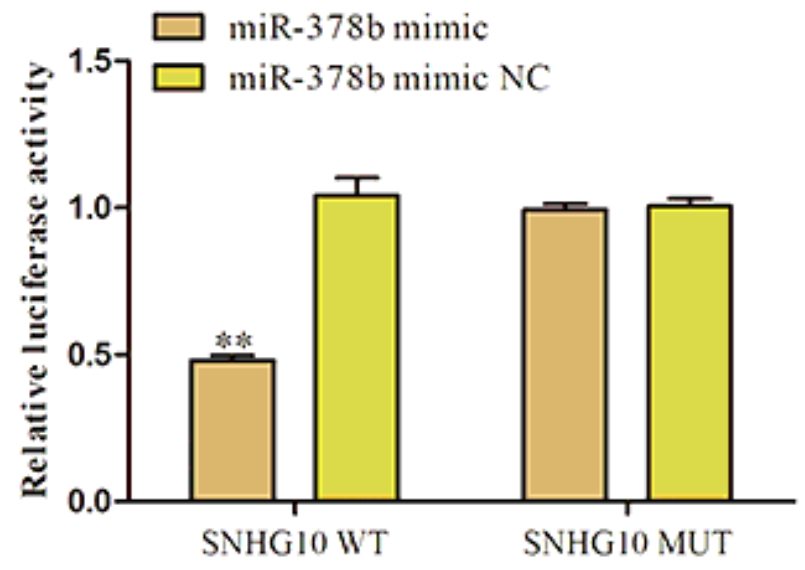

Figure 4

MiR-378b was a downstream target of SNHG10. (A) The binding site between SNHG10 and miR-378b were shown. $(B, C)$ The expression of miR-378b was detected by RT-PCR. ${ }^{\star} * \mathrm{P}<0.01$ vs. Blank or TGF- $\beta 1$. (D) Luciferase reporter assay was performed to verify the targeting relationship between SNHG10 and miR-378b. ${ }^{\star *} \mathrm{P}<0.01$ vs. Blank, TGF- $\beta 1$ or miR-378b mimic NC. 

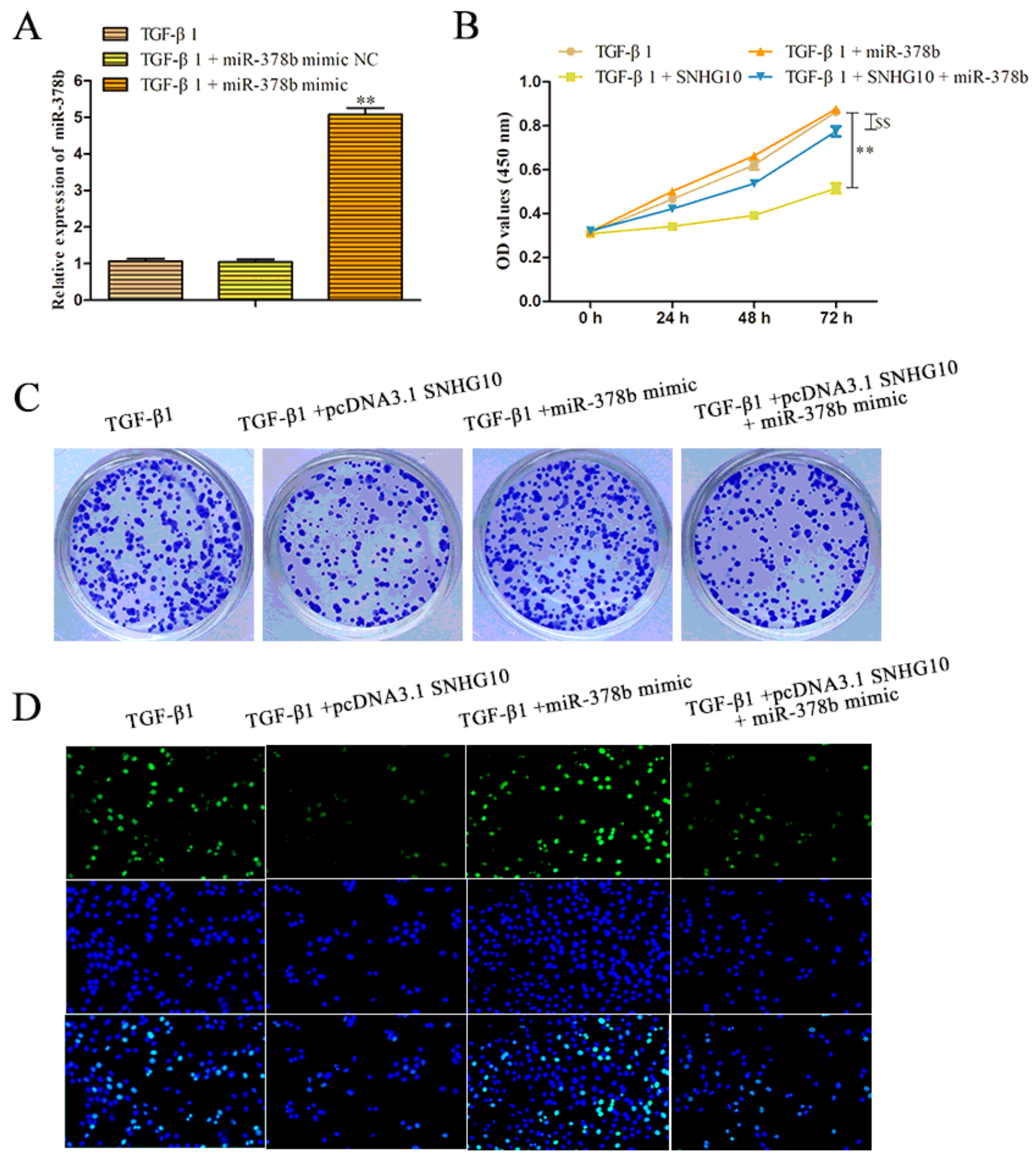

\section{Figure 5}

SNHG10 inhibits renal fibrosis through suppression of miR-378b expression. (A) The expression levels of miR-378b were measured to test the transfected efficiency. (B-D) Cell proliferation was measured by CCK8 , colony formation and EdU assays. All results were presented as mean \pm SD from at least three independent assay. ${ }^{* * P}<0.01$ vs. TGF- $\beta 1 . \$ \$ P<0.01$ vs. TGF- $\beta 1+$ pcDNA3.1 SNHG10. 

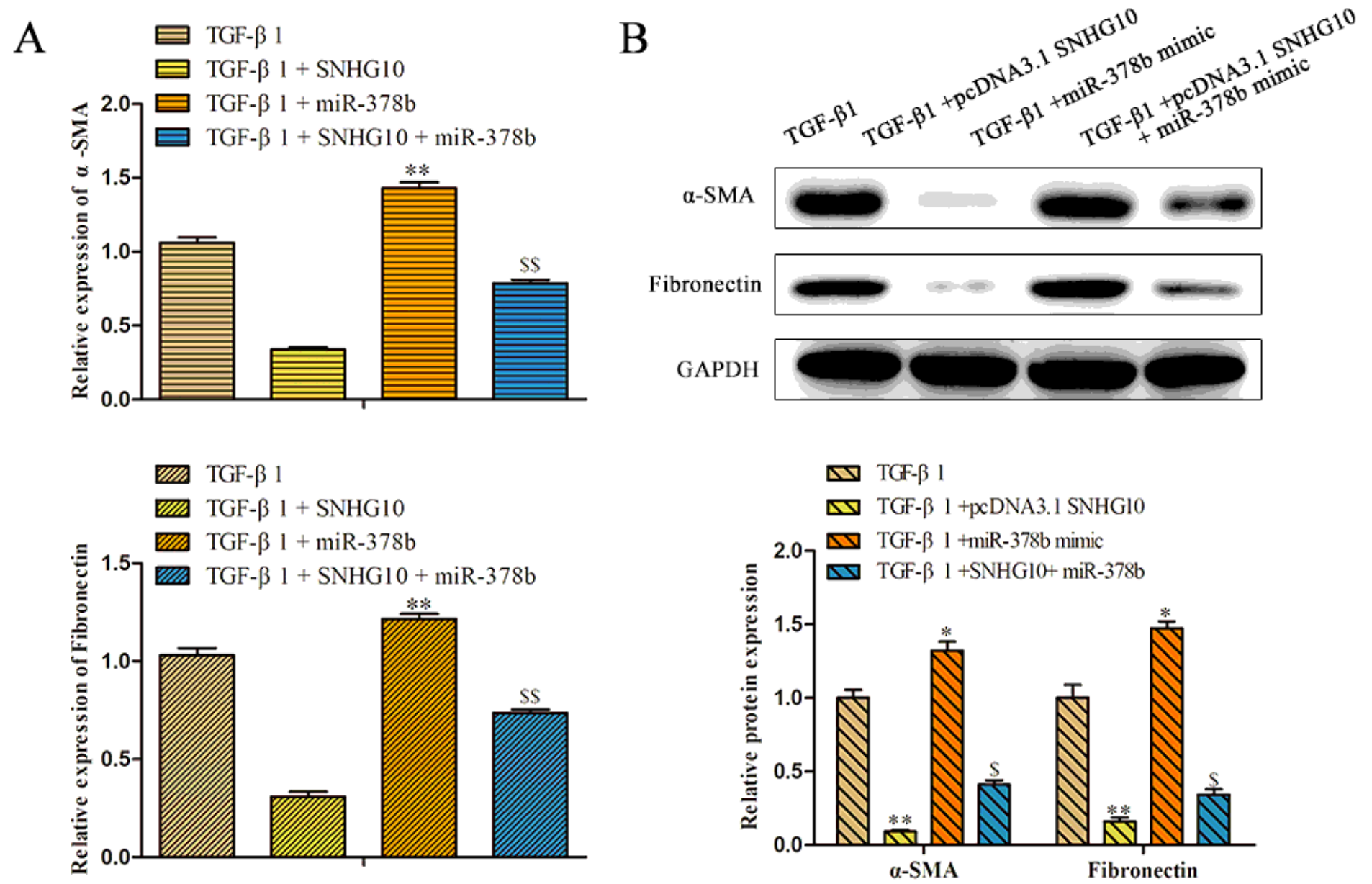

Figure 6

SNHG10 regulated renal fibrosis-associated markers through suppression of miR-378b expression. (A, B) qRT-PCR and western blot assays were applied to analyzed the mRNA and protein expression levels of aSMA and Fibronectins. ${ }^{*} P<0.01,{ }^{\star *} P<0.01$ vs. TGF- $\beta 1$. $\$ \mathrm{P}<0.05, \$ \$ \mathrm{P}<0.01$ vs. TGF- $\beta 1+$ pcDNA3.1 SNHG10. 